\title{
Tumores ováricos malignos en la infancia
}

Drs. Margarita Reyes, M. Inés Sins D. ${ }^{* *}$ A. Del Río, ${ }^{* * *}$ H. Del Pozo, ${ }^{* * * *}$ V. Beresi, ${ }^{* * *}$ J. J. Latorre.*****

\section{ABSTRACT}

\begin{abstract}
Nine cases of malignant ovarian tumors was registrted in the Oncology Service of the Luis Calvo Mackenna Hospital, from 1971 to July 1979.

Two of nine patients came from the principal city Santiago and seven cases were from other cities.

The most frequent reason of consultation was abdominal distention ar expantion.

In eight cases, the abdominal mass was clearly perceptible or palpable at the first physical examination.

The patients that cane from the other cities were operated before being reported to us.

In two sases, the first anatomopathological diasnostic was a benign Teratoma. But after the relapse of the tumor, the diagnosis was changed to malignant Teratoma.

The final diagmostic of the nine cases was based following the hystological classification proposed by the OMS, v.g. Innature Teratoma in seven cases; ovarian Disgerminoma in one case, and Tumor of the Senoendodemic in one case.

All the cases were classified in stages in accordance with FICO classification, Seven cases were in stages III and IV.

The treatment indicated was of differents types: Surgery, multiple chemiotherapy and radiotherapy.

Survival time was from 4 month to 4 years 5 month. Four of them died in this periode.
\end{abstract}

Los tumores ováricos malignos son una patología poco frecuente en la infancia. En estadisticas mundiales sólo constituyen el $1 \%$ de todas las neoplasias en niñas menores de 16 años. Por esto plantean al clínico problemas de diagnóstico, clasificación y conducta terapéutica. Se debe tener muy en cuenta que el tratamiento médico y quirúrgico de estos tumores, en este grupo etario, debe considerar cuanto sea posible el desarrollo de la niña y la conservación de la capacidad menstrual y reproductora de la futura mujer.

Es por todo esto que nos parecio de interés revisar la experiencia que al respecto tiene el Servicio de Oncología de nuestro hospital.

\section{MATERIAL Y METODO}

Se revisaron las fichas de los casos de tumor ovárico maligno que aparecen en el Libro de Registro del Servicio de Oncología del Hospital Luis Calvo Mackenna, que data del año 1968, encontrándose el ler caso de tumor ovárico en 1971. Hasta julio del presente año se recopilaron 9 casos.

Se analizó la edad de las pacientes, procedencia, síntomas y signos al consultar, diagnóstico de traslado, estado al ingresar a nuestro Servicio,

\footnotetext{
*Trabajo de Ingresa a la Socjedad de Pediatrfa.

** Jefe Depto. Ginecología Infantil y Adolescencia.

***Jefe Servicio de Cirugia Hospital Luis Calvo M.

**** Servicio Oncologia Hospital Luis Calvo M.

*****Jefe Depto. Anatomfa Patológica Hospital Luis Calvo M.
}

estudio que se le practicó en el mismo en base a exámenes de laboratorio y Anatomía Patológica, tratamiento y sobrevida.

Se clasificaron histológicamente según la clasificación propuesta por la OMS y en grados según la clasificación recomendada por la FIGO.

\section{CLASIFICACION HISTOLOGICA DE TUMO- RES OVARICOS HECHA POR LA ORGANIZA- CION MUNDIAL DE LA SALUD}
A. TUMORES EPITELIALES COMUNES.
1. Seroso.
2. Mucinoso.
3. Endometrioide.
4. De células claras (mesonefroide).
5. De Brenner.
6. Mixto.
7. Indiferenciado.
8. No clasificados.

\section{B. TUMORES DEL ESTROMA DE CORDO- NES SEXUALES}

1. Tumores de células de granulosa y de estroma:

a) Tumor de células de grarfulosa.

b) Grupo de tecoma-fibroma.
1. Tecoma.
2. Fibroma.

2. Androblastomas: tumores de células de Sertoli-Leydig.

3. Ginandroblastomas.

4. Noclasificados. 
C. TUMORES DE CELULAS LIPOIDES.

D. GONADOBLASTOMAS.

E. TUMORES DE CELULAS GERMINATIVAS:

1. Disgerminoma.

2. Tumor de seno endodérmico.

3. Carcinoma embrionario.

4. Poliembrioma.

5. Coriokarcinoma.

6. Teratomas:
a) Teratoma inmaduro.
b) Teratoma maduro.
c) Tumores monodérmicos y especializados.
7. Formas mixtas.

F. TRASTORNOS "CANCERIFORMES",

G. TUMORES METASTATICOS SECUNDARIOS.

\section{CLASIFICACION DE TUMORES OVARICOS KECOMENDADA POR LA FIGO.}

Etapa Ia. Tumor limitado a un ovario - sin ascitis.

Ib. Tumor limitado a ambos ovarios sin ascitis.

Ic. Tumor limitado a uno o ambos ovarios pero con ascitis o lavado peritoneal $(+)$.

Etapa IIa. Tumor ovárico con extensión a útero y lo trompas.

IIb. Con extensión a otros tejidos pelvianos.

IIc. Etapa IIa. o IIb., pero con ascitis o lavado peritoneal (+).
Etapa III. Turnor de uno o ambos ovarios con metástasis intraabdominal.

Etapa IV. Tumor de uno o ambos ovarios con metástasis a distancia fuera de la cavidad abdominal (pulmón, cerebro).

Nota: Si existe derrame pleural debe existir citología (t) en el líquido pleural para poder catalogarlo en Etapa IV.

Metástasis intrahepática automáticamente clasifican al tumor en Etapa IV.

\section{RESULTADOS}

De los 9 casos revisados, sólo 2 son de Santiago, los restantes fueron enviados de provincias: Rancagua, Curicó, 2 de Talca, Parral, Victoria y Puerto Varas.

La edad en el momento de consultar fluctuó en 4 años 8 meses y 13 años 4 meses, observándose un leve predominio en menores de 10 años.

\begin{tabular}{lll}
\hline Nombre & \multicolumn{1}{c}{ Edad } & Procedencia \\
\hline NT & 12años & Talca \\
MK & 13 años 4 meses & Puerto Varas \\
AM & 5 años 6 meses & Rancagua \\
MC & 11 años 1 mes & Curicó \\
MV & 12 años 2 meses & Santiago \\
FA & 8 años & Parral \\
AL & 9 años 7 meses & Santiago \\
VN & 7 años 11 meses & Victoria \\
VCH & 4 años 8 meses & Talea \\
\hline
\end{tabular}

DISTAIBUCION POA EDADES

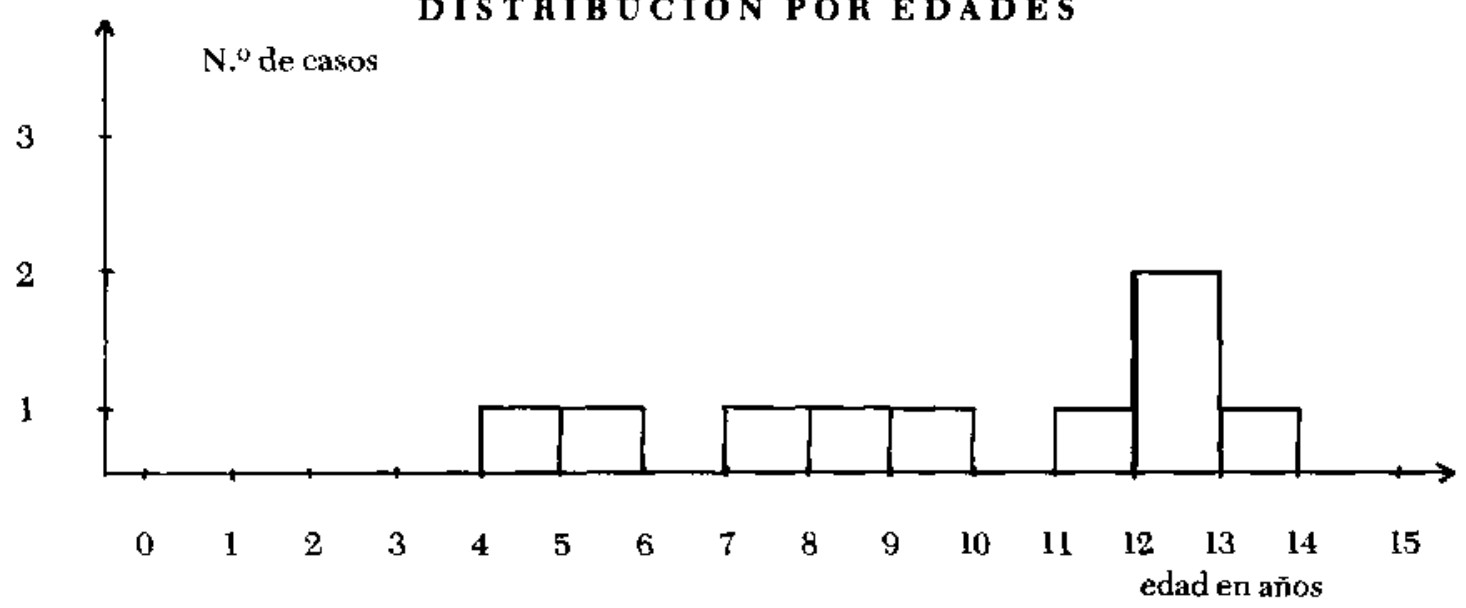


Los síntomas que motivaron la consulta, en la mayoría ( 6 casos), fueron por distensión y/o aumento del volumen abdominal. Ś́lo 3 por dolor abdominal, presentándose en 2 casos como cuadro agudo y agregándose adelgazamiento en 2 casos.

En cuanto al examen físico: en 8 pacientes se palpó la masa abdominal y en una fue un abdomen agudo. Se agregó palidez en 2 casos y gravedad en 1. A ninguno se le practicó examen ginecológico.

\section{MOTIVOS DE CONSULTA}

Distensión abdominal y/o aumento de

volumen abdominal

Dolor abdominal

Adelgazamiento

Cuadro Agudo

\section{EXAMEN FISICO}

Palpación de masa abdominal

Palidez

Grave

Abdomen Agudo

Sin examen ginecológico

De las 7 pacientes que venían de provincias, 6 habian sido intervenidas extirpándoseles el tumor ( 1 incluso reintervenida) y a la restante sólo se le practicó biopsia por considerarse el tumor inextirpable.

A 2 de estas niñas se les hizo el diagnóstico post-operatorio de teratoma benigno y ante recidiva del humor, una fue reintervenida y se llegó al diagnóstico de Teratoma maligno y en la otra se volvieron a revisar las placas concluyendo también en el diagnóstico de Teratoma maligno.

\section{OPERACION EN PROVINCIA}

\section{Extirpación Tumor}

Sólo biopsia

Reintervencion
Fueron enviadas a Santiago con los Diagnósticos de:

Teratoma maligno 5

Tumor de Willms

Cistoadenocarcinoma ovárico

A su ingreso al Servicio de Oncologia se comprobs que presentaban buen estado general $7 \mathbf{n i -}$ ñas y mal sólo 2; había 3 enflaquecidas y palidez en 2, taquipnea en 1 caso.

Al examen abdominal: en 6 pacientes habia aumento de volumen y masa palpable abdominal e incluso circulación colateral en 3 casos. En 3 niñas la palpación abdominal fue normal.

\section{EXAMEN FISICO INGRESO a ONCOLOGIA}

Buen estado general 7

$\mathrm{Mal}$ estado general

Enflaquecimiento

Palidez

Taquipnea

Aumento de volumen abdomen 6

Circulación colateral

Palpación masa abdominal

Abdomen normal

La evaluación de laboratorio nos mostró anemia en 2 pacientes y leucopenia sólo en 1 . La VHS estuvo alterada en 5 casos. Las alfa-fetoproteínas fueron + en 3 casos de los 7 a los cuales se les hizo el examen.

La Radiologia nos dio en 3 casos Radiografia de Tórax alterada; 1 derrame pleural, 1 proceso intersticial y 1 proceso agudo.

La Radiografía de abdomen simple nos mostró calcificaciones en 2 casos.

La Pielografía mostró dilatación de pelvis renal y rechazo de uréteres en 1 caso. Rechazo y comprensión extrínseca de uréteres en 2 casos.

La hadiografía de E.E.D. que se solicitó en un caso, reveló un desplazamiento de asas intestinales hacia arriba y a la izquierda.

\section{Segundo DG.}

Primer DG.

Post-operatorio

\begin{tabular}{|c|c|c|c|c|}
\hline MK & $\begin{array}{l}\text { Teratoma } \\
\text { benigno }\end{array}$ & Recidiva & $\begin{array}{l}\text { Reinter- } \\
\text { vención }\end{array}$ & $\begin{array}{l}\text { Teratoma } \\
\text { maligmo }\end{array}$ \\
\hline $\mathrm{V} \bar{X}$ & $\begin{array}{l}\text { Teratoma } \\
\text { benigno }\end{array}$ & Recidiva & $\begin{array}{l}\text { Hevisión } \\
\text { placas }\end{array}$ & $\begin{array}{l}\text { Teratoma } \\
\text { maligno }\end{array}$ \\
\hline
\end{tabular}




\section{EXAMENES DE LABORATORIO}

INGRESO A ONCOLOGIA

\begin{tabular}{lccc} 
& Alterado & Nonnal & $\begin{array}{c}\text { No se hizo o } \\
\text { no consignado } \\
(-)\end{array}$ \\
$\begin{array}{l}\text { Anemia } \\
\text { (Menos de } 10 \text { g de } \mathrm{Hb} \text { ) }\end{array}$ & 2 & 7 & $(-)$ \\
Leucopenia (Menos de 4.000) & 1 & 8 & 2 \\
VHS (sobre 20) & 5 & 2 & 2 \\
Alfa Fetoproteínas (+a+++) & 3 & 4 & 1 \\
RX Tórax & 3 & 5 & 6 \\
RX Abdomen simple & 2 & 1 & 6 \\
Pielografía & 3 & $(-)$ & 8 \\
RX E.E.D. & 1 & $(-)$ & \\
\hline
\end{tabular}

Los diagnósticos histopatológicos se hicieron en base a biopsia practicada en el Servicio en 5 casos; revisión de placas enviadas de provincias en 3 casos y en 1 caso por necropsia y los diagnosticos fueron:

$\begin{array}{ll}\text { Teratoma ovárico inmaduro } & 7 \\ \text { Disgerminoma } & 1 \\ \text { Tumor Senoendodérmico } & 1\end{array}$

La clasificación en Etapas clínicas fue:

Etapa I a 1

Etapa II b 1

Etapa III 4

Etapa IV 3

El tratamiento que recibieron fue quirúrgico, qumioterapia y radioterapia.

En 4 casos no se hizo tratamiento quirúrgico: ya habían sido operadas en provincias. En un caso se practicó sólo biopsia por considerarse inextir- pable la recidiva tumoral. En otro caso se extirpó el tumor recidivado. En otro, se extirpó el tumor recidivado más oofoorectomía contralateral y resección de sigmoides por adherencias.

De las 2 pacientes de Santiago que no habian sido operadas, a una se le practicó extirpación del tumor y de metástasis en diafragma, epiplón y Douglas; a la otra se le extirpó el tumor, la mitad de la trompa del mismo lado, el apéndice y se tomó biopsia del Douglas.

Quimioterapia se les hizo a todas las pacientes, recibiendo distintas asociaciones de drogas. La más usada fue VACM.

Radioterapia se aplicó en 5 casos, sobre pelvis en dosis que fueron de 2.000 a 4.600 rads.

Sobrevida: De las 9 pacientes han fallecido 4 , en períodos de 1 mes a 1 año 5 ms.

En las restantes, la sobrevida va de 4 años a 5 ms.

TRATAMIENTO EN SERVICIO DE ONCOLOGIA

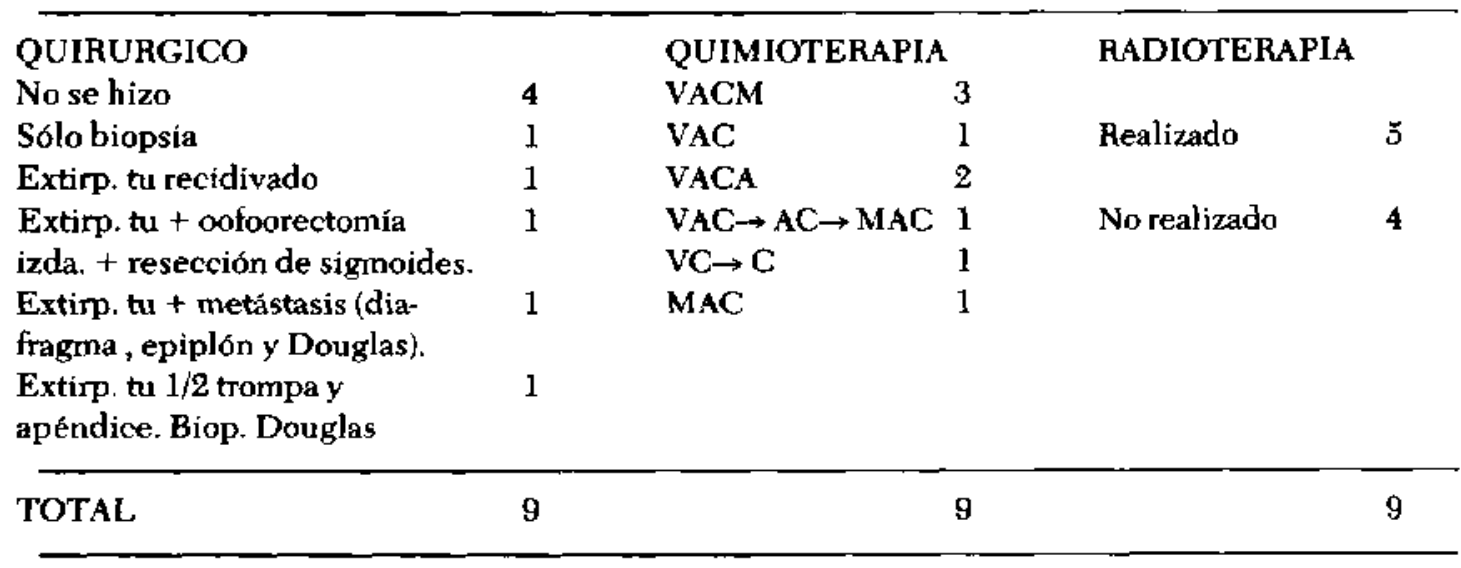




\begin{tabular}{|c|c|c|c|c|c|c|}
\hline Nombre & Diagnóstico & Etapa & $\begin{array}{l}\text { Tratamiento } \\
\text { Quirúrgico }\end{array}$ & $\begin{array}{l}\text { Quimio- } \\
\text { terapia }\end{array}$ & $\begin{array}{l}\text { Radio- } \\
\text { terapia }\end{array}$ & $\begin{array}{c}\text { Sobrevida } \\
\text { a VIL-79 }\end{array}$ \\
\hline NT & Teratoma ovárico inmaduro & IV & $(-)$ & VACM & $(-)$ & $+1 \mathrm{~ms}$. \\
\hline MK & Teratoma ovárico inmaduro $\mathrm{OP}$ & III & $(-)$ & VACM & $(+)$ & +1 a $5 \mathrm{~ms}$ \\
\hline $\mathbf{A M}$ & Teratoma ovárico inmaduro $O P$ & III & $(-)$ & VAC & $(-)$ & +5 ms. \\
\hline $\mathbf{M C}$ & Tumor senoendodérnico OP & $\mathrm{IV}$ & Biopsia & $\mathrm{VC} \rightarrow \mathrm{C}$ & $(-)$ & $+2 \mathrm{~ms}$ \\
\hline MV & Disgerminoma ovárico & IIb & $\begin{array}{l}\text { TU + 1/2 } \\
\text { Trompa + } \\
\text { Apéndice }+ \\
\text { Biop. Douglas }\end{array}$ & $\begin{array}{l}\mathrm{VAG} \rightarrow \mathrm{AC} \rightarrow \\
\mathrm{MAC} .\end{array}$ & $\begin{array}{l}(+) \\
(+)\end{array}$ & 4 a $5 \mathrm{~ms}$. \\
\hline FA & Teratoma ovárico inmaduro OP & III & TU recidivado & MAC & $(+)$ & 3 a 5 ms. \\
\hline AL & Teratoma ovárico inmaduro & IV & $\begin{array}{l}\text { TU y Metás- } \\
\text { tasis (Diafrag- } \\
\text { ma, Epiplón } \\
\text { y Douglas }\end{array}$ & VACM & $(+)$ & 1 a $8 \mathrm{~ms}$. \\
\hline $\mathrm{VN}$ & Teratoma ovárico inmaduro OP & III & $\begin{array}{l}\text { TU + oofoo- } \\
\text { rectomia izq. } \\
\text { resección } \\
\text { sigmoides }\end{array}$ & VACA & $(+)$ & 1 a $5 \mathrm{~ms}$. \\
\hline VCH & Teratoma ovárico inmaduro OP & $\mathbf{I} \mathbf{a}$ & $(-)$ & VACA & $(-)$ & $4 \mathrm{~ms}$. \\
\hline
\end{tabular}

\section{CONCLUSIONES}

De acuerdo a lo que hemos revisado podemos concluir: En cuanto a la edad en que se presentan este tipo de tumores, no hubo lactantes ni menores de 4 años. E] disgerminoma y el tumor del senoendodérmico se presentaron en nif́as mayores de 10 años.
En cuanto al principal motivo de consulta fue aumento de volumen y/o distensión abdominal.

Al examen, en casi la totalidad de los casos, se palpó una masa abdominal y no pélvica. La ascitis no constituyó un elemento tan importante de malignidad como ocurre en las mujeres adultas.

En cuanto a los diagnósticos de tumos benigno en 2 casos, que posteriormente se debió cam-

Distribución por edades
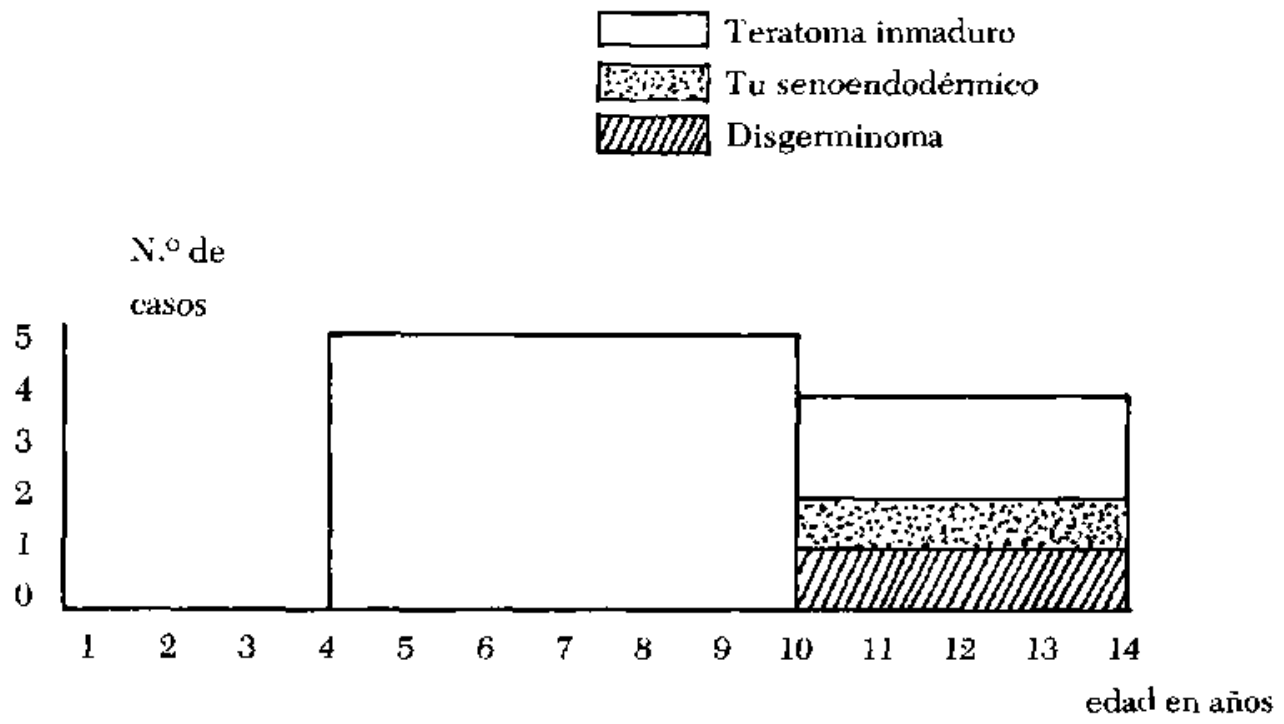
biar por el de turnor maligno ante recidiva, esto no es raro que ocurra en los teratomas, y las publicaciones internacionales al respecto, así lo señalan y recomiendan un minimo de 50 cortes para tener un diagnóstico histopatológico seguro.

Los exámenes de laboratorio que son de apoyo diagnóstico claro serian: VHS, alfafetoproteinas cuando son $(+)$ y el estudio radiográfico en base a radiografía de abdomen simple y pielografía, fundamentalmente.

En cuanto a los diagnósticos definitivos todos los tumores pertenecían a tumores de células germinativas, coincidiendo con revisiones mundiales que destacan un franco predominio en las niñas de los tumores de células germinales en relación a los de células epiteliales, que son mucho más frecuentes en la mujer adulta. Casi todas las estadísticas dan mayor frecuencia a los disgerminomas que a los teratomas inmaduros entre los tumores germinales. Sin embargo, en nuestra revisión el predominio franco es de los teratomas inmaduros.

En cuanto al tratamiento, la cirugía fue conservadora, pero no debemos olvidar que venfan en su mayoría operadas y se encontraban en etapas III y IV.

En cuanto a la Quimioterapia las drogas que se asocian han sido diferentes a través del tiempo, siendo opinión del Servicio, en este momento, que la más apropiada sería la de Vincristina + Actinomicina D + Ciclofosfamida + Adriamicina, por cuanto es la que tiene mayor posibilidad de exito y menor toxicidad hematológica, gastroin-. testinal o neurotóxica.

La Radioterapia fue útil unida a la cirugia y quimioterapia que fue el tratamiento de elección en el caso de disgerminoma. Esta paciente tiene una sobrevida actual de 4 años 5 ms. y 16 años 7 ms. de edad y se encuentra en tratamiento estrogénico sustitutivo tendiente a estimular su desarollo y presencia de caracteres sexuales secundarios.

Como conclusion final diremos que este tema es de enfoque multidisciplinario; en él participan el oncólogo, ginecólogo, cirujano, quimioterapeuta, radioterapeuta, radiólogo, anatomopatólogo además del pediatra general que recibe a estas pacientes en el consultorio o en el Servicio de Urgencia por alguna complicación.

Deseamos por último mostrarles cuál es nuestra conducta actual a seguir en estos tumores de acuerdo a la clasificación de la FIGO.
Etapa I b : Exéresis del tumor

Quimioterapia

Etapa I b : Panhisterectomía - Omentectomía Apendicectomja

Quimioterapia

Etapa I c : Panhisterectomía - Omentectomf́a Apendicectomía

Radioterapia a la pelvis

Quimioterapia

Etapa LIa, IIb y IIc :

Panhisterectomía - Omentectomia Apendicectomía

Biopsia de ganglios inguinales $y$ lumboaórticos

Radioterapia a la pelvis

Quimioterapia

Etapa III : Quirúrgico (la mayor extirpación razonablemente posible del tumor).

Quimioterapia fundamentalmente

Radioterapia como tratamiento coadyuvante

Etapa IV : El tratamiento fundamental es la quimioterapia, reservando la cirugía como coadyuvante.

\section{RESUMEN}

Se presentan 9 casos de humor ovárico maligno, que son los registrados en el Servicio de Oncologia desde 1971 hasta julio del presente año. La edad de las pacientes va desde 4 a 13 años.

De los 9 casos, 2 eran de Santiago y 7 de provincia.

El motivo de consulta más frecuente fue distensión y/o aumento de volumen abdominal.

Al primer examen físico casi todas (8) presentaron masa abdominal palpable.

Las pacientes de provincia fueron todas laparatomizadas antes de ser referidas. En 2 de ellas se hizo el diagnóstico anátomo-patológico en primera instancia de Teratoma benigno y ante recidiva del tumor se llegó al diagnóstico de Teratoma maligno.

En el Servicio de Oncología se llegó a los diagnósticos definitivos según la clasificación histologica propuesta por la OMS de: Teratoma inmaduroen 7 casos. Disgerminoma ovárico, 1 caso. Tumor del Senoendodérmico, 1 caso. Fueron clasificados en etapas según la clasificación de la F1GO, encontrándose la mayoria de ellos (7) en etapas III y IV.

En cuanto al tratamiento éste fue asociado: cirugía, quimioterapia múltiple y radioterapia. 
La sobrevida va de 4 meses a 4 años 5 meses y con 4 pacientes fallecidas.

\section{REFERENCIAS}

lFor, $C h$. Adnexal malignancy detected by cervical cytology". Am J. Obstet. Gynecol J32 (2): 143-50, 15 Sep. 1978.

${ }^{2}$ Curry, S. Lu et al. "Malignant teratoma of the ovary: pronostic factors and treatment", Am J. Obstet Gynecol 131 (B): 845-9, Aug. 1978.

${ }^{3}$ Kanalf $e t$ al. "The develnpment of a double-antibody radioinmunoassay for detecting ovarian tumor - associated antigen fraction OCA in plasma. "Am J. Obstet Gynecol 131 ( 7 ); 780-7, (1) August. 1978.

${ }^{4}$ Piver, M S. al. "Incidence of subclinical metastasis in stage I and II Ovarian carcinoma". Obstet Gynecol 52 (1): 100-4. Jul. 1978.

5julian, G. G. et al, "Radionctive phosphorus and extemal radiation as an adjuvant to surgery for ovarian carcinoma". Obstet Gyaceol 52 (2): 155-60. Aug. 1978.

${ }^{6}$ Slayton, R. E. et al. "Treatment of malignant ovarian germ cel tumors: response to vincristine, dactinomycin and cyclophosphamide" (preliminary report). Cancer 42 (2): 390-8, Aug. 1978.

${ }^{7}$ Pall, B. Underwood, J. R. and Kreutner, J. R. "Neoplasms and Tumors Conditions of the ovary and Tube". Adolescent obstetrics and Gymecology: 503-530. 1978.

${ }^{B}$ Huffman, W.: "The Gynecology of Chilhood and Adoles. cence". (Philadelphia: W. B. Saunders Company, 1968).

${ }^{G}$ Breen, J. L. y Maxon, W. S. "Tumores Ováricos en Niñas y Adolescentes". Cilnicas Obstêtricas y Gínecologicas: 515-637, Sept. 1977. 